\title{
High resolution spectroscopy over 8500-8750 Å for GAIA ${ }^{\star, \star \star, \star \star \star}$
}

\section{A library of synthetic spectra for $7750 \leq T_{\text {eff }} \leq 50000 \mathrm{~K}$}

\author{
F. Castelli ${ }^{1}$ and U. Munari ${ }^{2,3}$ \\ 1 CNR-GNA-Osservatorio Astronomico di Trieste Via Tiepolo 11, 34131 Trieste, Italy \\ 2 Osservatorio Astronomico di Padova, Sede di Asiago, 36012 Asiago (VI), Italy \\ 3 Centro Interpardimentale Studi ed Attività Spaziali (CISAS "G. Colombo"), Università di Padova, Italy
}

Received 8 September 2000 / Accepted 23 November 2000

\begin{abstract}
In this paper we complete the library of synthetic spectra for the range $7650-8750 \AA$, which includes the 8500-8750 A interval currently base-lined for the spectroscopic observations by GAIA, candidate ESA Cornerstone 5 mission. As for Paper II, the spectra are based on Kurucz's codes and line data. The explored metallicity, gravity and temperature ranges are $-2.5 \leq\left[Z / Z_{\odot}\right] \leq+0.5,4.5 \leq \log g \leq 2.0$ and $7750 \leq T_{\text {eff }} \leq 50000 \mathrm{~K}$, respectively. The 698 new spectra are computed at the same $\lambda / \triangle \lambda=20000$ resolving power of the observed spectra given in Paper I (131 standard stars mapping the MKK spectral classification system) and the 254 synthetic spectra of Paper II (characterized by $T_{\text {eff }} \leq 7500 \mathrm{~K}$ ).
\end{abstract}

Key words. atlases - surveys - stars: fundamental parameters

\section{Introduction}

GAIA is the approved ESA Cornerstone 6 mission designed to obtain extremely precise astrometry (in the micro-arcsec regime), multi-band photometry and medium/high resolution spectroscopy for a large sample of stars. The goals as depicted in the mission Red Book (ESA SP-2000-4) call for astrometry and broad band photometry to be collected for all stars down to $V \sim 20$ mag over the entire sky ( $\sim 110^{9}$ stars), with brighter magnitude limits for spectroscopy and intermediate band photometry. Each target star should be measured over a hundred times during the five year mission life-time, in a fashion similar to the highly successful Hipparcos operational mode. The astrophysical guidelines of the GAIA mission are discussed by Gilmore et al. (1998) and Perryman (1999), an overview of the GAIA payload and spacecraft is presented

Send offprint requests to: U. Munari,

e-mail: munari@pd.astro.it

* Tables 2-4 are only available in electronic form at the CDS via anonymous ftp to cdsarc.u-strasbg.fr (130.79.128.5) or via

http://cdsweb.u-strasbg.fr/cgi-bin/qcat?J/A+A/366/1003 or via the personal HomePage

http://ulisse.pd.astro.it/Astro/Atlases/

$\star \star$ The spectra are available in electronic form at the CDS.

$\star \star \star$ Figures 5-224 are only available in electronic form at http://www . edpsciences.org by Mérat et al. (1999), and the goals of the GAIA spectroscopy and the merits of the $8500-8750 \AA$ region are discussed by Munari (1999).

This third paper completes the establishment of a data-bank of input data for simulations and evaluation of GAIA spectroscopic performances. As for the whole series, the results are also of general interest to ground-based spectroscopists working at moderately high resolving powers in the near-IR region of the spectrum. Throughout this series a constant resolving power $R=\lambda / \triangle \lambda=20000$ has been maintained. This is at the higher end of the range of dispersions currently considered for implementation on GAIA (between 0.25 and $1.00 \AA /$ pix). Observing and computing at the highest resolution leaves unaltered the possibilities to degrade the results to a lower resolution as later possibly required, while the contrary is not feasible.

In Paper I (Munari \& Tomasella 1999) we have built up a homogeneous observational data-bank composed by the spectra of 131 standard stars mapping the MKK classification system from types O4 to M8 and luminosity classes from I to V. In Paper II (Munari \& Castelli 2000) we presented a library of 254 synthetic spectra covering the part of the $\left[Z / Z_{\odot}\right], \log g$ and $T_{\text {eff }}$ space where the vast majority of GAIA targets will be located. They are F-G-K-M type stars with metallicities ranging from those of the galactic globular clusters to that of the Pop. I objects and thus the spectral grid extended over $-2.5 \leq\left[Z / Z_{\odot}\right] \leq+0.5,4.5 \leq \log g \leq 1.0$ and 

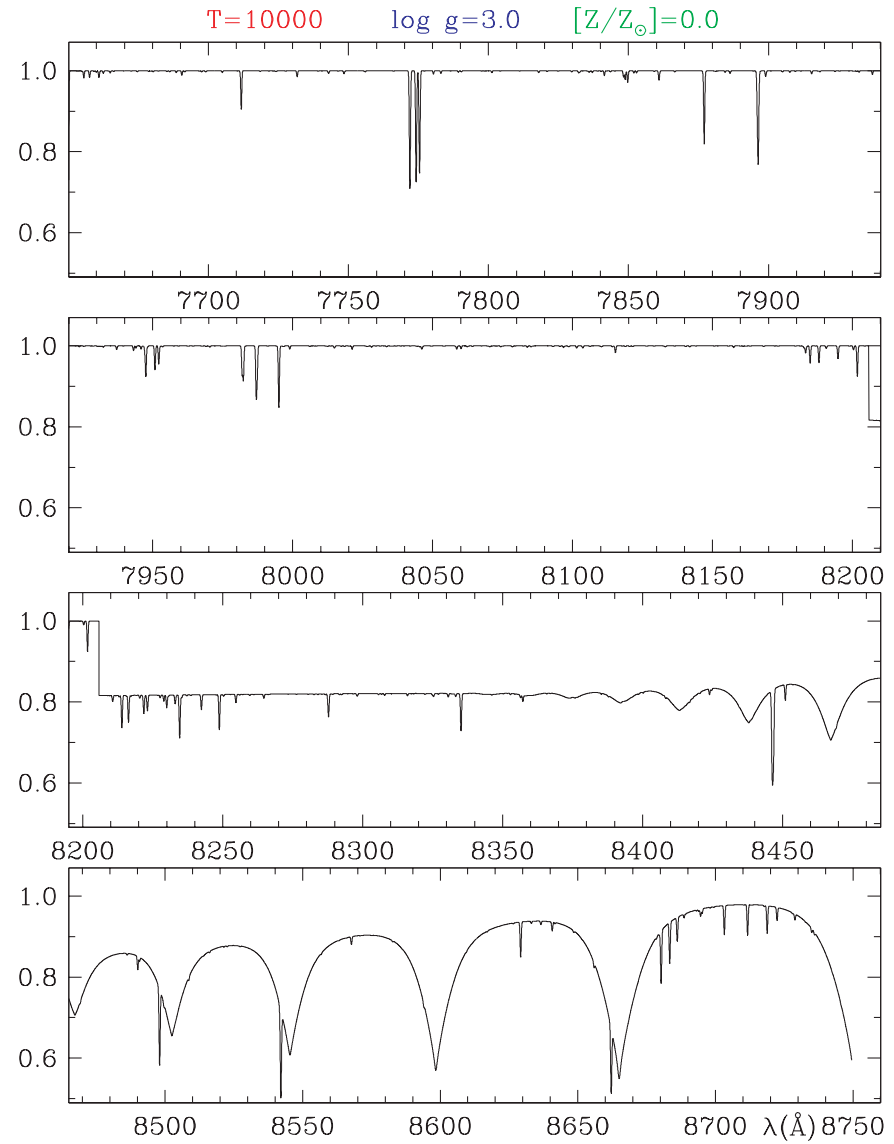

Fig. 1. All spectra have been computed over a range wider that the $8500-8750 \AA$ considered in this series of papers. This plot shows the full explored range $(7650-8750 \AA)$

$T_{\text {eff }} \leq 7500 \mathrm{~K}$. The present Paper III completes the spectral mapping toward the higher temperatures (covering $\mathrm{A}, \mathrm{B}$ and $\mathrm{O}$ type stars) by presenting 698 new spectra in the $7750 \mathrm{~K} \leq T_{\text {eff }} \leq 50000 \mathrm{~K}$ temperature range.

\section{The synthetic spectra}

The grid of 698 computed synthetic spectra is listed in Table 1 where the number of the electronic figure containing the spectra is given. In fact, for each $T_{\text {eff }},\left[Z / Z_{\odot}\right]$ pair, there is a figure which displays spectra for different surface gravities which roughly correspond to luminosity classes V, III, and I in the MKK classification system. Only the pair of spectra corresponding to $T_{\text {eff }}=32000 \mathrm{~K}$, $\left[Z / Z_{\odot}\right]=+0.5, \log g=3.25$ and $T_{\text {eff }}=40000 \mathrm{~K}$, $\left[Z / Z_{\odot}\right]=+0.5, \log g=4.0$ are missing in this scheme (cf. Table 1 and Figs. 214 and 224), because no radiative equilibrium for the upper layers of the two models was ever achieved in the computations.

The spectra for the whole 7650-8750 $\AA$ interval (including the spectra of Paper I and Paper II) are available in electronic ASCII form at the CDS. An example of computed spectrum normalized to the continuum level for the full $7650-8750 \AA$ interval and $T_{\text {eff }}=10000 \mathrm{~K},\left[Z / Z_{\odot}\right]=$ $0.0, \log g=3.0$ parameters is presented in Fig. 1 . All the other Figs. 2-224 show the computed spectrum normal-

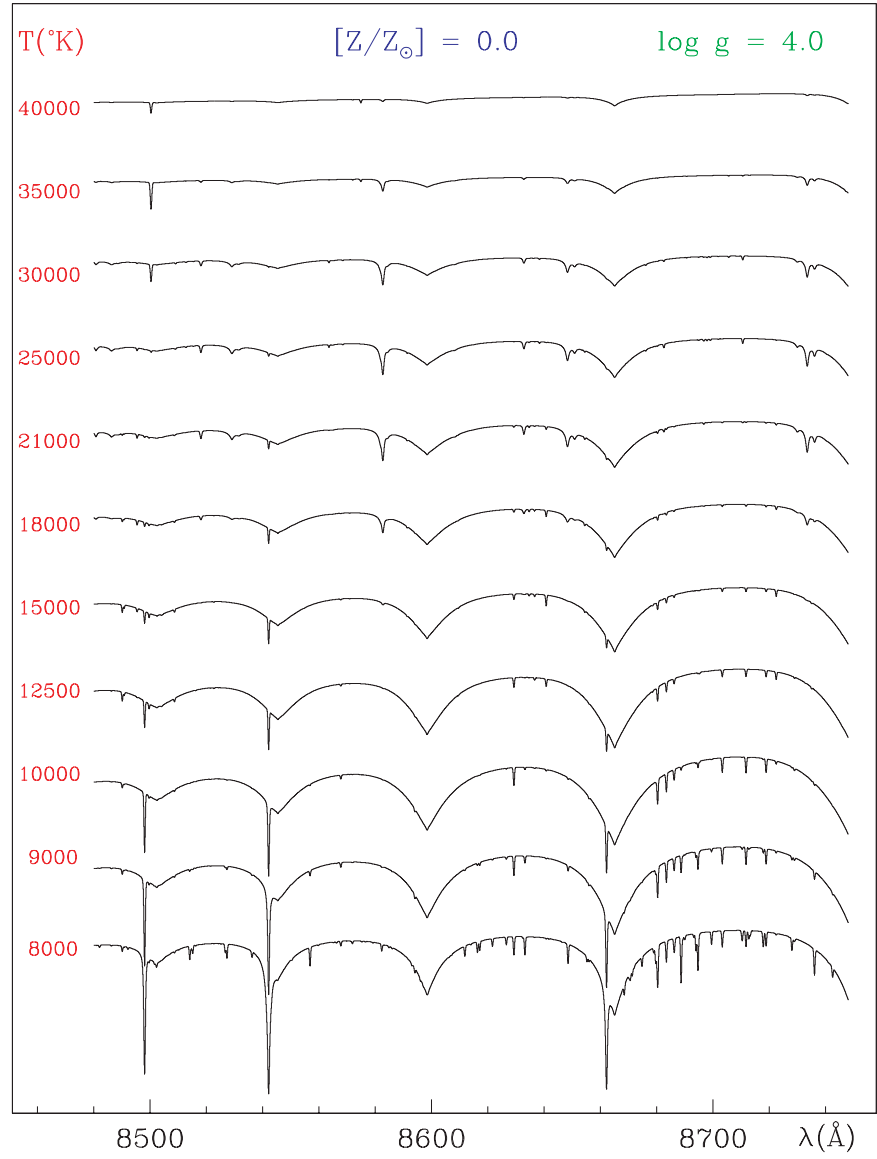

Fig. 2. A sample of $\left[Z / Z_{\odot}\right]=0.0$ synthetic spectra arranged in a sequence showing the effect of varying the temperature

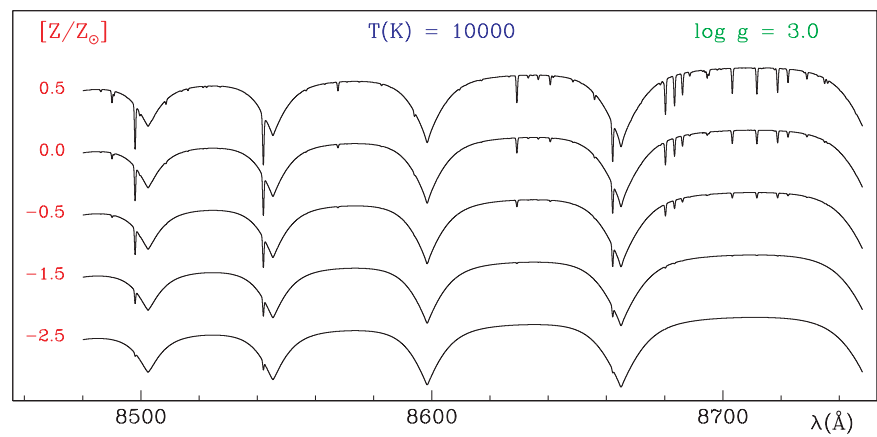

Fig. 3. A sample of $T_{\text {eff }}=10000 \mathrm{~K}, \log g=3.0$ synthetic spectra arranged in a sequence showing the effect of varying the metallicity

ized to the continuum level focusing on the GAIA 8500$8750 \AA$ interval only. To avoid over-crowding in Figs. 220 224 , the spectra with $T_{\text {eff }}=37500 \mathrm{~K}, \log g=4.5$ and $T_{\text {eff }}=34000 \mathrm{~K}, \log g=4.5$ are not plotted.

The effects caused by changing the temperature, the metallicity and the gravity in a few example cases are illustrated in Figs. 2, 3 and 4 respectively. Figure 4 is a template for the Figs. 5-224 only available in electronic form. 
Table 1. The Metallicity-Temperature-Gravity grid mapped by our synthetic spectra. The numbers-in-the-boxes give the corresponding electronic figure where the given triplet of spectra is plotted. For the numbers in bracketts see text, Sect. 2

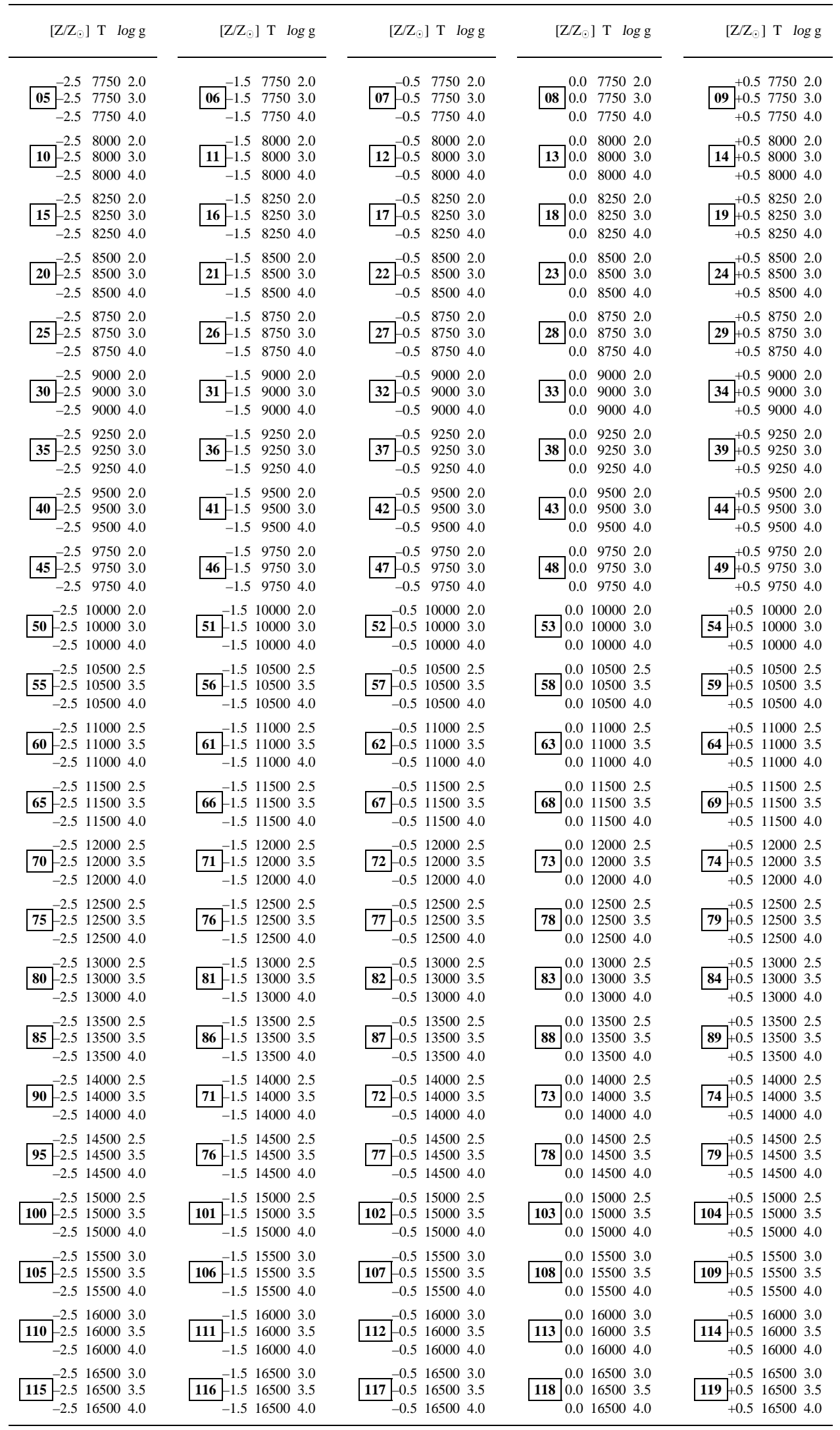


Table 1. continued

\begin{tabular}{|c|c|c|c|c|c|c|c|c|c|c|c|c|}
\hline & {$\left[\mathrm{Z} / \mathrm{Z}_{\odot}\right] \mathrm{T} \quad 1$} & $\log \mathrm{g}$ & {$\left[\mathrm{Z} / \mathrm{Z}_{\odot}\right] \mathrm{T} \quad l$} & $\log g$ & & {$\left[\mathrm{Z} / \mathrm{Z}_{\odot}\right] \mathrm{T}$} & $\log \mathrm{g}$ & & {$\left[\mathrm{Z} / \mathrm{Z}_{\odot}\right] \mathrm{T}$} & $\log g$ & {$\left[\mathrm{Z} / \mathrm{Z}_{\odot}\right] \mathrm{T}$} & $\log g$ \\
\hline \multirow{3}{*}{120} & $-2.5 \quad 17000$ & 3.0 & $-1.5 \quad 17000$ & 3.0 & & $-0.5 \quad 17000$ & 3.0 & & 0.017000 & 3.0 & $+0.5 \quad 17000$ & 3.0 \\
\hline & $-2.5 \quad 17000$ & 3.5 & \begin{tabular}{|l|ll}
$\mathbf{1 2 1}$ & -1.5 & 17000
\end{tabular} & 3.5 & 122 & $-0.5 \quad 17000$ & 3.5 & 123 & 0.017000 & 3.5 & \begin{tabular}{|l|ll}
$\mathbf{1 2 4}+0.5$ & 17000
\end{tabular} & 3.5 \\
\hline & -2.517000 & 4.0 & $-1.5 \quad 17000$ & 4.0 & & $-0.5 \quad 17000$ & 4.0 & & 0.017000 & 4.0 & +0.517000 & 4.0 \\
\hline \multirow{3}{*}{125} & -2.517500 & 3.0 & -1.517500 & 3.0 & & $-0.5 \quad 17500$ & 3.0 & & 0.017500 & 3.0 & $\sigma^{+0.5 \quad 17500}$ & 3.0 \\
\hline & -2.517500 & 3.5 & \begin{tabular}{|lll}
$\mathbf{1 2 6}$ & -1.5 & 17500
\end{tabular} & 3.5 & \begin{tabular}{|l|}
127 \\
\end{tabular} & $\begin{array}{lll}-0.5 & 17500\end{array}$ & 3.5 & 128 & 0.017500 & 3.5 & \begin{tabular}{|lll}
$\mathbf{1 2 9}+0.5$ & 17500
\end{tabular} & 3.5 \\
\hline & -2.517500 & 4.0 & $-1.5 \quad 17500$ & 4.0 & & $-0.5 \quad 17500$ & 4.0 & & 0.017500 & 4.0 & $+0.5 \quad 17500$ & 4.0 \\
\hline & -2.518000 & 3.0 & -1.518000 & 3.0 & & $-0.5 \quad 18000$ & 3.0 & & 0.018000 & 3.0 & +0.518000 & 3.0 \\
\hline \multirow{3}{*}{130} & -2.518000 & 3.5 & \begin{tabular}{|l|ll}
$\mathbf{1 3 1}$ & -1.5 & 18000 \\
\end{tabular} & 3.5 & 132 & $-0.5 \quad 18000$ & 3.5 & 133 & 0.018000 & 3.5 & \begin{tabular}{|lll}
$\mathbf{1 3 4}+0.5$ & 18000
\end{tabular} & 3.5 \\
\hline & -2.518000 & 4.0 & $-1.5 \quad 18000$ & 4.0 & & $-0.5 \quad 18000$ & 4.0 & & 0.018000 & 4.0 & $+0.5 \quad 18000$ & 4.0 \\
\hline & -2.518500 & 3.0 & -1.518500 & 3.0 & & $-0.5 \quad 18500$ & 3.0 & & 0.018500 & 3.0 & +0.518500 & 3.0 \\
\hline \multirow{3}{*}{135} & $-2.5 \quad 18500$ & 3.5 & \begin{tabular}{|lll}
$\mathbf{1 3 6}$ & -1.5 & 18500
\end{tabular} & 3.5 & \begin{tabular}{|l|l}
137 & \\
\end{tabular} & $-0.5 \quad 18500$ & 3.5 & 138 & 0.018500 & 3.5 & $\mathbf{1 3 9}+0.518500$ & 3.5 \\
\hline & -2.518500 & 4.0 & $-1.5 \quad 18500$ & 4.0 & & $-0.5 \quad 18500$ & 4.0 & & 0.018500 & 4.0 & $+0.5 \quad 18500$ & 4.0 \\
\hline & -2.519000 & 3.0 & $-1.5 \quad 19000$ & 3.0 & & $-0.5 \quad 19000$ & 3.0 & & 0.019000 & 3.0 & $+0.5 \quad 19000$ & 3.0 \\
\hline \multirow[t]{3}{*}{140} & -2.519000 & 3.5 & \begin{tabular}{|l|l|l|l|l|}
141 & 19000 \\
\end{tabular} & 3.5 & 142 & $-0.5 \quad 19000$ & 3.5 & 143 & 0.019000 & 3.5 & \begin{tabular}{|ll}
$\mathbf{1 4 4}+0.5$ & 19000
\end{tabular} & 3.5 \\
\hline & -2.519000 & 4.0 & $-1.5 \quad 19000$ & 4.0 & & $-0.5 \quad 19000$ & 4.0 & & 0.019000 & 4.0 & +0.519000 & 4.0 \\
\hline & -2.519500 & 3.0 & $-1.5 \quad 19500$ & 3.0 & & $-0.5 \quad 19500$ & 3.0 & & 0.019500 & 3.0 & $+0.5 \quad 19500$ & 3.0 \\
\hline \multirow[t]{2}{*}{145} & -2.519500 & 3.5 & $\mathbf{1 4 6}-1.519500$ & 3.5 & 147 & $-0.5 \quad 19500$ & 3.5 & 148 & 0.019500 & 3.5 & $\mathbf{1 4 9}+0.519500$ & 3.5 \\
\hline & -2.519500 & 4.0 & -1.519500 & 4.0 & & $-0.5 \quad 19500$ & 4.0 & & 0.019500 & 4.0 & $+0.5 \quad 19500$ & 4.0 \\
\hline \multirow{3}{*}{150} & -2.520000 & 3.0 & -1.520000 & 3.0 & & $-0.5 \quad 20000$ & 3.0 & & 0.020000 & 3.0 & +0.520000 & 3.0 \\
\hline & -2.520000 & 3.5 & 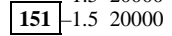 & 3.5 & 152 & $-0.5 \quad 20000$ & 3.5 & 153 & 0.020000 & 3.5 & \begin{tabular}{|ll}
$\mathbf{1 5 4}+0.520000$ \\
\end{tabular} & 3.5 \\
\hline & -2.520000 & 4.0 & -1.520000 & 4.0 & & -0.520000 & 4.0 & & 0.020000 & 4.0 & +0.520000 & 4.0 \\
\hline \multirow{3}{*}{155} & -2.521000 & 3.0 & -1.521000 & 3.0 & & $-0.5 \quad 21000$ & 3.0 & & 0.021000 & 3.0 & +0.521000 & 3.0 \\
\hline & -2.521000 & 3.5 & \begin{tabular}{|lll}
156 & -1.5 & 21000
\end{tabular} & 3.5 & \begin{tabular}{|l|l|}
157 \\
\end{tabular} & $-0.5 \quad 21000$ & 3.5 & 158 & 0.021000 & 3.5 & \begin{tabular}{|l|ll}
$\mathbf{1 5 9}+0.5$ & 21000
\end{tabular} & 3.5 \\
\hline & -2.521000 & 4.0 & -1.521000 & 4.0 & & -0.521000 & 4.0 & & 0.021000 & 4.0 & +0.521000 & 4.0 \\
\hline & -2.522000 & 3.0 & -1.522000 & 3.0 & & $-0.5 \quad 22000$ & 3.0 & & 0.022000 & 3.0 & +0.522000 & 3.0 \\
\hline 160 & -2.522000 & 3.5 & \begin{tabular}{|l|ll}
$\mathbf{1 6 1}$ & -1.5 & 22000 \\
\end{tabular} & 3.5 & 162 & $-0.5 \quad 22000$ & 3.5 & 163 & 0.022000 & 3.5 & \begin{tabular}{|lll}
$\mathbf{1 6 4}+0.522000$ &
\end{tabular} & 3.5 \\
\hline & -2.522000 & 4.0 & -1.522000 & 4.0 & & $-0.5 \quad 22000$ & 4.0 & & 0.022000 & 4.0 & +0.522000 & 4.0 \\
\hline & -2.523000 & 3.0 & -1.523000 & 3.0 & & $-0.5 \quad 23000$ & 3.0 & & 0.023000 & 3.0 & +0.523000 & 3.0 \\
\hline 165 & -2.523000 & 3.5 & $\begin{array}{llll}\mathbf{1 6 6} & -1.5 & 23000\end{array}$ & 3.5 & \begin{tabular}{|l|l|}
167 & \\
\end{tabular} & $-0.5 \quad 23000$ & 3.5 & 168 & 0.023000 & 3.5 & \begin{tabular}{|lll}
$\mathbf{1 6 9}+0.5$ & 23000
\end{tabular} & 3.5 \\
\hline & -2.523000 & 4.0 & -1.523000 & 4.0 & & $-0.5 \quad 23000$ & 4.0 & & 0.023000 & 4.0 & +0.523000 & 4.0 \\
\hline & -2.524000 & 3.0 & $-1.5 \quad 24000$ & 3.0 & & -0.524000 & 3.0 & & 0.024000 & 3.0 & +0.524000 & 3.0 \\
\hline 170 & -2.524000 & 3.5 & $\mathbf{1 7 1}-1.524000$ & 3.5 & 172 & -0.524000 & 3.5 & 173 & 0.024000 & 3.5 & $\mathbf{1 7 4}+0.524000$ & 3.5 \\
\hline & -2.524000 & 4.0 & -1.524000 & 4.0 & & $-0.5 \quad 24000$ & 4.0 & & 0.024000 & 4.0 & +0.524000 & 4.0 \\
\hline & -2.525000 & 3.0 & $-1.5 \quad 25000$ & 3.0 & & $-0.5 \quad 25000$ & 3.0 & & 0.025000 & 3.0 & $+0.5 \quad 25000$ & 3.0 \\
\hline 175 & -2.525000 & 3.5 & 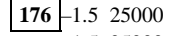 & 3.5 & 177 & $-0.5 \quad 25000$ & 3.5 & 178 & 0.025000 & 3.5 & \begin{tabular}{|l|l|}
$179+0.5$ & 25000 \\
\end{tabular} & 3.5 \\
\hline & -2.525000 & 4.0 & $-1.5 \quad 25000$ & 4.0 & & $-0.5 \quad 25000$ & 4.0 & & 0.025000 & 4.0 & +0.525000 & 4.0 \\
\hline & -2.526000 & 3.0 & -1.526000 & 3.0 & & -0.526000 & 3.0 & & 0.026000 & 3.0 & $+0.5 \quad 26000$ & $3.0(59)$ \\
\hline \begin{tabular}{|l|}
180 \\
\end{tabular} & -2.526000 & 3.5 & \begin{tabular}{|lll}
181 & -1.5 & 26000
\end{tabular} & 3.5 & 182 & $-0.5 \quad 26000$ & 3.5 & 183 & 0.026000 & 3.5 & $\mathbf{1 8 4}+0.526000$ & 3.5 \\
\hline & -2.526000 & 4.0 & -1.526000 & 4.0 & & $-0.5 \quad 26000$ & 4.0 & & 0.026000 & 4.0 & +0.526000 & 4.0 \\
\hline & -2.527000 & 3.0 & -1.527000 & 3.0 & & $-0.5 \quad 27000$ & 3.0 & & 0.027000 & $3.0(63)$ & $+0.5 \quad 27000$ & $3.0(59)$ \\
\hline 185 & -2.527000 & 3.5 & \begin{tabular}{|ll}
186 & -1.5 \\
27000
\end{tabular} & 3.5 & \begin{tabular}{|l|l|}
187 & \\
\end{tabular} & $-0.5 \quad 27000$ & 3.5 & 188 & 0.027000 & 3.5 & \begin{tabular}{|l|ll}
$\mathbf{1 8 9}+0.5$ & 27000
\end{tabular} & 3.5 \\
\hline & -2.527000 & 4.0 & -1.527000 & 4.0 & & -0.527000 & 4.0 & & 0.027000 & 4.0 & +0.527000 & 4.0 \\
\hline & -2.528000 & 3.0 & -1.528000 & 3.0 & & $-0.5 \quad 28000$ & 3.0 & & 0.028000 & $3.0(54)$ & +0.528000 & $3.0(57)$ \\
\hline 190 & -2.528000 & 3.5 & \begin{tabular}{|l|l|l|}
191 & -1.5 & 28000
\end{tabular} & 3.5 & 192 & $-0.5 \quad 28000$ & 3.5 & 193 & 0.028000 & 3.5 & \begin{tabular}{|l|l|}
$\mathbf{1 9 4}+0.528000$ & 280
\end{tabular} & 3.5 \\
\hline & -2.528000 & 4.0 & -1.528000 & 4.0 & & -0.528000 & 4.0 & & 0.028000 & 4.0 & +0.528000 & 4.0 \\
\hline & -2.529000 & 3.0 & -1.529000 & 3.0 & & $-0.5 \quad 29000$ & $3.0(57)$ & & 0.029000 & $3.0(54)$ & +0.529000 & $3.25(57)$ \\
\hline 195 & -2.529000 & $\begin{array}{l}3.5 \\
4.0\end{array}$ & \begin{tabular}{|l|ll}
196 & -1.5 & 29000 \\
-1.5 & 29000
\end{tabular} & 3.5 & 197 & $-0.5 \quad 29000$ & $\begin{array}{l}3.5 \\
4.0\end{array}$ & 198 & 0.029000 & 3.5 & 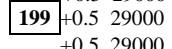 & \\
\hline & -2.530000 & 3.25 & -1.530000 & 3.25 & & -0.530000 & 3.25 & & 0.030000 & $3.25(63)$ & +0.530000 & $3.25(57)$ \\
\hline 200 & -2.530000 & 3.5 & \begin{tabular}{|l|l|l|l|}
201.5 & 30000
\end{tabular} & 3.5 & 202 & -0.530000 & 3.5 & 203 & 0.030000 & & $2 \mathbf{2 0 4}+0.530000$ & \\
\hline & -2.530000 & 4.0 & -1.530000 & 4.0 & & -0.530000 & 4.0 & & 0.030000 & 4.0 & +0.530000 & 4.0 \\
\hline & -2.531000 & 3.25 & -1.531000 & 3.25 & & -0.531000 & 3.25 & & 0.031000 & $3.25(53)$ & +0.531000 & $3.25(58)$ \\
\hline 205 & -2.531000 & 3.5 & $206-1.531000$ & 3.5 & \begin{tabular}{|l|}
207 \\
\end{tabular} & -0.531000 & 3.5 & 208 & 0.031000 & 3.5 & $209+0.531000$ & $3.5(58)$ \\
\hline & -2.531000 & 4.0 & -1.531000 & 4.0 & & -0.531000 & 4.0 & & 0.031000 & 4.0 & +0.531000 & 4.0 \\
\hline & -2.532000 & 3.25 & -1.532000 & 3.25 & & -0.532000 & $3.25(57)$ & & 0.032000 & 3.25( & & \\
\hline 210 & -2.532000 & 3.5 & 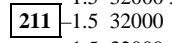 & 3.5 & 212 & -0.532000 & 3.5 & 213 & ] 0.032000 & & 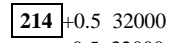 & $3.5(58)$ \\
\hline & -2.532000 & 4.0 & -1.532000 & 4.0 & & -0.532000 & 4.0 & & 0.032000 & 4.0 & +0.532000 & 4.0 \\
\hline 215 & -2.533000 & 3.5 & 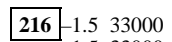 & 3.5 & \begin{tabular}{|l|}
217 \\
\end{tabular} & -0.533000 & 3.5 & 218 & 0.033000 & 3.5 & 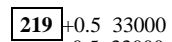 & $3.5(56)$ \\
\hline & -2.533000 & 4.0 & -1.533000 & 4.0 & & -0.533000 & 4.0 & & 0.033000 & & +0.533000 & 4.0 \\
\hline & $\begin{array}{l}-2.534000 \\
-2.534000\end{array}$ & $\begin{array}{l}3.5 \\
4.0\end{array}$ & $\begin{array}{l}-1.534000 \\
-1.534000\end{array}$ & $\begin{array}{l}3.5 \\
4.0\end{array}$ & & $\begin{array}{r}-0.534000 \\
-0.534000\end{array}$ & $\begin{array}{l}3.5 \\
4.0\end{array}$ & & $\begin{array}{l}0.034000 \\
0.034000\end{array}$ & $\begin{array}{l}3.5(53) \\
4.0\end{array}$ & $\begin{array}{l}+0.534000 \\
+0.534000\end{array}$ & 4.5 \\
\hline & -2.535000 & 3.5 & -1.535000 & 3.5 & & $-0.5 \quad 35000$ & 3.5 & & $\begin{array}{l}.0 .034000 \\
0.03500\end{array}$ & $3.5(47)$ & $\begin{array}{r}+0.534000 \\
+0.535000\end{array}$ & 4.0 \\
\hline & -2.535000 & 4.0 & -1.535000 & 4.0 & & -0.535000 & 4.0 & & 0.035000 & & +0.535000 & 4.5 \\
\hline 220 & -2.537500 & 4.0 & 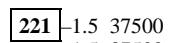 & 4.0 & 222 & -0.537500 & 4.0 & 223 & 0.037500 & 4.0 & 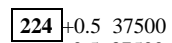 & 4.0 \\
\hline & -2.537500 & 4.5 & -1.537500 & 4.5 & & -0.537500 & 4.5 & & 0.037500 & 4.5 & +0.537500 & \\
\hline & -2.540000 & 4.0 & -1.540000 & 4.0 & & -0.540000 & 4.0 & & 0.040000 & $4.0(55)$ & & \\
\hline & $\begin{array}{l}-2.540000 \\
-2.5\end{array}$ & 4.5 & $\begin{array}{l}-1.540000 \\
-5\end{array}$ & 4.5 & & -0.540000 & 4.5 & & 0.040000 & 4.5 & +0.540000 & 4.5 \\
\hline & $\begin{array}{l}-2.542500 \\
-25.45000\end{array}$ & 4.5 & $\begin{array}{l}-1.542500 \\
-15\end{array}$ & 4.5 & & -0.542500 & 4.5 & & 0.042500 & 4.5 & $\begin{array}{r}+0.542500 \\
+054500\end{array}$ & $\begin{array}{l}4.5(61) \\
45.56\end{array}$ \\
\hline & $\begin{array}{l}-2.545000 \\
-2.547500\end{array}$ & $\begin{array}{l}4.5 \\
4.5\end{array}$ & $\begin{array}{l}-1.545000 \\
-1.547500\end{array}$ & $\begin{array}{l}4.5 \\
4.5\end{array}$ & & $\begin{array}{l}-0.545000 \\
-0.547500\end{array}$ & 4.5 & & $\begin{array}{l}0.045000 \\
0.047500\end{array}$ & $\begin{array}{l}4.5 \\
4.5\end{array}$ & $\begin{array}{r}+0.545000 \\
+0.547500\end{array}$ & $\begin{array}{l}4.5(56) \\
4.5(56)\end{array}$ \\
\hline & -2.550000 & 4.5 & $\begin{array}{l}-1.54 \\
-1.5 \\
-50000\end{array}$ & 4.5 & & $-0.5 \quad 50000$ & 4.5 & & $\begin{array}{l}.0 .04 / 500 \\
0.050000\end{array}$ & $4.5(57)$ & $\begin{array}{l}+0.545000 \\
+0.550000\end{array}$ & $4.5(56)$ \\
\hline
\end{tabular}




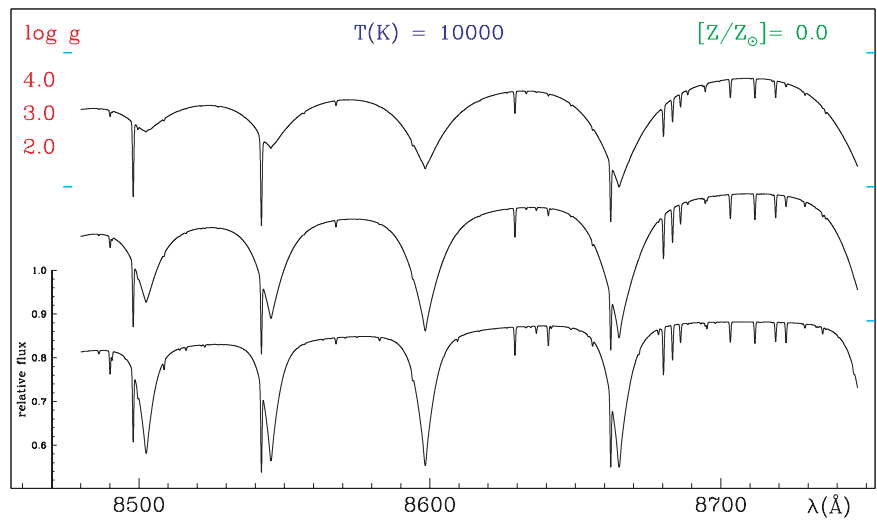

Fig. 4. A sample of $T_{\text {eff }}=10000 \mathrm{~K},\left[Z / Z_{\odot}\right]=0.0$ synthetic spectra arranged in a sequence showing the effect of varying the gravity. The thick dashes mark the 1.00 level of the continuum. This is an example of the Figs. 5-224 available only electronically

The most relevant absorption lines in the 8490-8750 range are tabulated in Table 2 for $T_{\text {eff }}=8000 \mathrm{~K}(\log g=$ 2.0 and 4.0$)$ and $T_{\text {eff }}=11000 \mathrm{~K}(\log g=2.5$ and 4.0$)$, in Table 3 for $T_{\text {eff }}=15000 \mathrm{~K}(\log g=2.5$ and 4.0) and $T_{\text {eff }}=20000 \mathrm{~K}(\log g=3.0$ and 4.0$)$, in Table 4 for $T_{\text {eff }}=26000 \mathrm{~K}(\log g=3.0$ and 4.0$), T_{\text {eff }}=33000 \mathrm{~K}$ $(\log g=3.5$ and 4.0$)$, and $T_{\text {eff }}=40000 \mathrm{~K}(\log g=4.5)$.

The synthetic spectra are based on the grids of model atmospheres computed by Kurucz (1993a). Only for $T_{\text {eff }} \leq 8750 \mathrm{~K}$ the models are from Castelli (1999) and differ from Kurucz's models only for the convection. Most of the models used in this paper are available at Kurucz's website (http: //kurucz . harvard. edu). Models not available there were computed by us with the ATLAS9 code. However, some of the additional models computed for high $T_{\text {eff }}$ and low gravity may be not accurate enough owing to the too small number of layers used for their computations. This shortcoming is related with the lack of line opacity data for small pressures $\left(\log P_{\text {gas }}<-2.0\right)$ in the opacity distribution function tables we used (Kurucz $1993 \mathrm{c}-\mathrm{e})$. Because the gas pressure is very low in the uppermost layers of hot giants, the inadequate opacity gives rise to numerical problems which we have overcame by dropping the upper layers. The number of adopted layers is indicated in Table 1 in parenthesis for models computed with less than 64 optical depths, which is the minimum number of layers used in the Kurucz's grids.

The synthetic spectra were computed with the SYNTHE code of Kurucz (1993b) at a 500000 resolving power. The spectra were degraded to the $\lambda / \Delta \lambda=20000$ resolving power by adopting a Gaussian instrumental profile. The line spectrum was computed for a microturbulent velocity $\xi=2 \mathrm{~km} \mathrm{~s}^{-1}$, while no rotational velocity and no macroturbulent velocity have been considered.

For the synthetic spectra we adopted the solar abundances given by Grevesse et al. (1996) while the model atmospheres (which are based on Kurucz' ODFs) use the Anders \& Grevesse (1989) solar abundances. Elements for which the abundances in model atmospheres and synthetic spectra differ are $\mathrm{N}(-0.08) \mathrm{O}(-0.06), \mathrm{F}(-0.04), \mathrm{S}(+0.09)$, $\mathrm{Ar}(-0.06), \mathrm{K}(-0.12)$, Sc $(+0.08)$, Ti $(+0.04)$, Fe $(-0.15)$, $\mathrm{Sr}(+0.07), \mathrm{Zr}(+0.10)$, La $(-0.05)$, Ce $(+0.03)$. The numbers in parenthesis are the logarithmic differences between the abundances from Anders \& Grevesse and Grevesse et al. (1996). Finally, Kurucz \& Bell (1995) has been the source for the atomic lines data (see also Kurucz 1995 and the Kurucz's website).

\section{References}

Anders, E., \& Grevesse, N. 1989, Geochim. Cosmochim. Acta, 53,197

Black, J. H., Wisheit, J. C., \& Laviana, E. 1972, ApJ, 177, 567

Castelli, F. 1999, A\&A, 346, 564

Friedrich, H., \& Trefftz, E. 1969, MPI-PAE/Astro, 29

Fuhr, J. R., Martin, G. A., \& Wiese, W. L. 1988., J. Phys. Chem. Ref. Data, 17, Suppl. 4

Gilmore, G., Perryman, M., Lindegren, L., et al. 1998, Proc. SPIE Conf., 3350, 541

Grevesse, N., Noels, A., \& Sauval, A. J. 1996, in Cosmic Abundances, ed. S. S. Holt, \& G. Sonneborn, ASP Conf. Ser., 99, ASP San Francisco, 117

Kurucz, R. L. 1988, Trans. IAU, XXB, ed. M. McNally (Kluwer), 168

Kurucz, R. L. 1993a, ATLAS9 Stellar Atmospheres Programs and $2 \mathrm{~km} \mathrm{~s}^{-1}$ grid, CD-ROM, No. 13

Kurucz, R. L. 1993b, SYNTHE Spectrum Synthesis Programs and Line Data, CD-ROM, No. 18

Kurucz, R. L. 1993c, Opacities for Stellar Atmospheres: [+0.0], $[+0.5],[+1.0]$, CD-ROM No. 2

Kurucz, R. L. 1993d, Opacities for Stellar Atmospheres: [-0.5], $[-1.0],[-1.5]$, CD-ROM No. 3

Kurucz, R. L. 1993e, Opacities for Stellar Atmospheres: [-2.0], [-2.5], [-3.0], CD-ROM No. 4

Kurucz, R. L. 1995, Astrophysical Applications of Powerful New Databases, ed. S. J. Adelman, \& W. L. Wiese, ASP Conf., 78, 205

Kurucz, R. L., \& Bell, B. 1995, Atomic Line List, CD-ROM, No. 23

Kurucz, R. L., \& Peytremann, E. 1975, SAO Special Report, 362

McEachran, R. P., \& Cohen, M. 1971, JQSRT, 11, 1819

Merat, P., Safa, F., Camus, J. P., Pace, O., \& Perryman, M. A. C. 1999, in Proceedings of the ESA Leiden Workshop on GAIA, 23-27 Nov. 1998, Baltic Astron., 8, 1

Munari, U. 1999, in Proceedings of the ESA Leiden Workshop on GAIA, 23-27 Nov. 1998, Baltic Astron., 8, 73

Munari, U., \& Tomasella, L. 1999, A\&AS, 137, 521 (Paper I)

Munari, U., \& Castelli, F. 2000, A\&AS, 141, 141 (Paper II)

Murphy, P. W. 1968, JOSA, 58, 1200

Perryman, M. A. C. 1999, S\&T, 97, 48

Wiese, W. L., Smith, M. W., \& Glennon, B. M. 1966, NSRDSNBS 4 